\title{
OPTICAL LABEL RECOGNITION BASED ON ADDITIONAL PRE-SPREAD CODING
}

\author{
Hideaki Furukawa, ${ }^{1}$ Tsuyoshi Konishi, ${ }^{1}$ Kazuyoshi Itoh, ${ }^{1}$ \\ Naoya Wada, ${ }^{2}$ and Fumito Kubota ${ }^{2}$ \\ ${ }^{1}$ Graduate School of Engineering, Osaka University, 2-1, Yamadaoka, Suita, Osaka 565-0871, \\ Japan, ${ }^{2}$ National Institute of Information and Communications Technology, Tokyo, Japan
}

\begin{abstract}
We propose novel optical label recognition based on additional pre-spread coding. This method can realize both high recognition power in label recognition and data transmission efficiency in network paths. We present the optical implementation of this method and show the recognition power by simulation.
\end{abstract}

\section{INTRODUCTION}

In the next-generation photonic networks based on a packet switch, optical label recognition is a key technology to route optical packets according to label without any electronic bottlenecks. Since an optical correlation system can recognize temporal optical codes only by passive optical devices without a complex synchronization, it is considered to be a powerful solution for optical label recognition [1-4]. In an optical label recognition system based on optical correlation, the recognition can be accomplished by comparing the maximum intensity of correlation signals between optical codes and a matched filter. To improve its recognition power, it is desirable that the maximum intensity ratio (SNR : signal to noise ratio) of correlation signals of the target optical code to non-target ones is high. Since the bit-pattern of long-bit spread codes are generally much different from each other, spread codes, such as M-sequences or Gold codes, are used as optical codes to raise the SNR [2,4]. However, since long-bit spread codes occupy large part of packets, data transmission efficiency becomes much low in network paths.

In the network, it is said that the number of labels of $10^{4}$ is required [2]. Generally, the bit length of optical codes is 14-bits enough to represent labels of $10^{4}$. However, 256-bits or more are necessary for spread codes to represent labels of $10^{4}$ [4]. From the viewpoint of data transmission efficiency, it is 
desirable that the bit length of transmitted optical codes is as short as possible in network paths. Here, if transmitted short-bit optical codes can be expanded into long-bit spread codes just before label recognition in network nodes, both high SNR and high data transmission efficiency can be realized. In this paper, we propose optical label recognition based on additional pre-spread coding.

\section{OPTICAL LABEL RECOGNITION USING ADDITIONAL PRE-SPREAD CODING SYSTEM}

Figure 1 shows the conceptual diagram of the proposed optical label recognition based on additional pre-spread coding. In network paths, short-bit optical codes are used to achieve high data transmission efficiency. In network nodes, these short-bit optical codes are expanded into long-bit spread codes by an additional pre-spread coding system. After the additional pre-spread coding, since the obtained long-bit spread codes are much different from each other, high SNR can be achieved in the label recognition based on optical correlation.

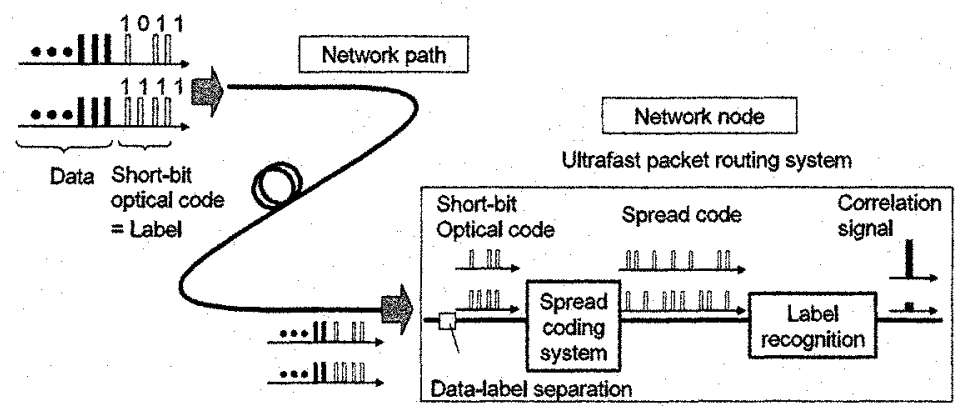

Figure 1. Optical label recognition using additional pre-spread coding.

Here, we adopt Gold codes as one of spread codes to realize the proposed additional pre-spread coding system. The generating procedure of Gold codes is shown in Fig. 2. First, M-sequence 1 is generated from an input short-bit optical code. M-sequence 2 is an arbitrary M-sequence, which is prepared in advance. Secondly, a Gold code is generated by executing EXOR operation between $M$-sequence 1 and 2. Here, a set of $M$-sequences can be generated from the initial bit-pattern of M-sequence 1 by shifting it with different amount depending on each input short-bit optical code. By executing EXOR operation between this set of M-sequences (for example M1-001, M1-010, and so on) and M-sequence 2, we can obtain a set of Gold codes from different input short-bit optical codes. Here, there is a feature that M-sequence of a short-bit optical code can be generated by executing EXOR operation between M-sequences 
generated from each bit of the optical code. For example, M-sequence of [011] can be obtained by executing EXOR operation between that of [001] and [010]. Thus, we have only to construct an optical setup which generates M-sequence from each bit and executes EXOR operation for spread coding.

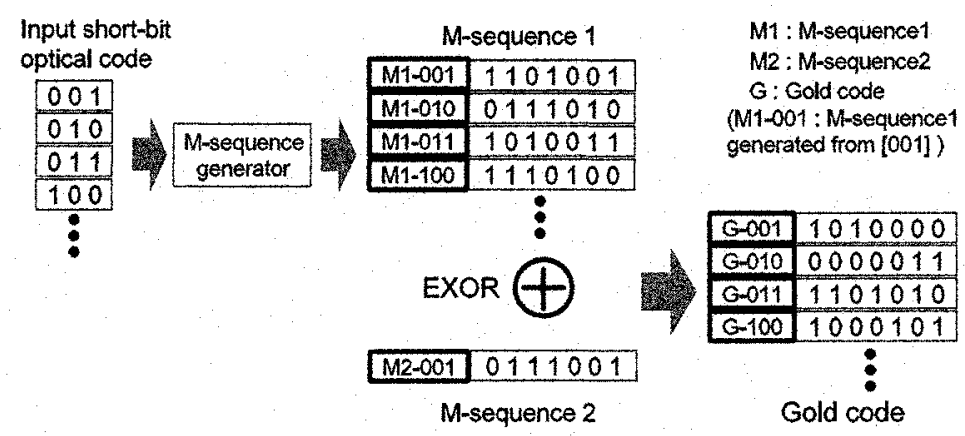

Figure 2. Generation procedure of Gold code.

The optical setup of spread coding system is shown in Figs. 3 and 4. As shown in Fig. 3a, an arbitrary M-sequence can be generated from one bit by adequately setting fiber delay lines in the M-sequence generator. In case of several bits amplitude shift keying (ASK) optical codes, M-sequences are generated from each bit by this generator. (Fig. 3b.) The summation of these M-sequences is output as the M-sequence 1 of several bits optical codes. Moreover, the $\mathrm{M}$-sequence 1 is added to $\mathrm{M}$-sequence 2 generated from one clock signal. Instead of directly executing EXOR operation between these $M$-sequences, we achieve equivalent processing to EXOR by using this summation and the intensity conversion. As shown in Fig. 4a, to realize equivalent processing to EXOR, the intensity of optical pulses is converted into 0 or 1 at the even or odd intensity level respectively. The intensity conversion is implemented using optical multilevel thresholding [5]. In the high nonlinear fiber (HNLF), self-frequency shifting generates, and the center frequency of optical pulses is shifted depending on the intensity. (Fig. 4b.) As a result, each optical pulse can be separated by arrayed waveguide grating (AWG). (Fig. 4c.) The intensity level of each optical pulse is adjusted to 0 or 1 by appropriate filters. Consequently, ASK Gold codes can be generated by this setup. This method can also obtain binary phase shift keying (BPSK) Gold codes by converting the intensity level of each pulse into 1 and modulating the phase to 0 or $\pi$ with phase shifters. 


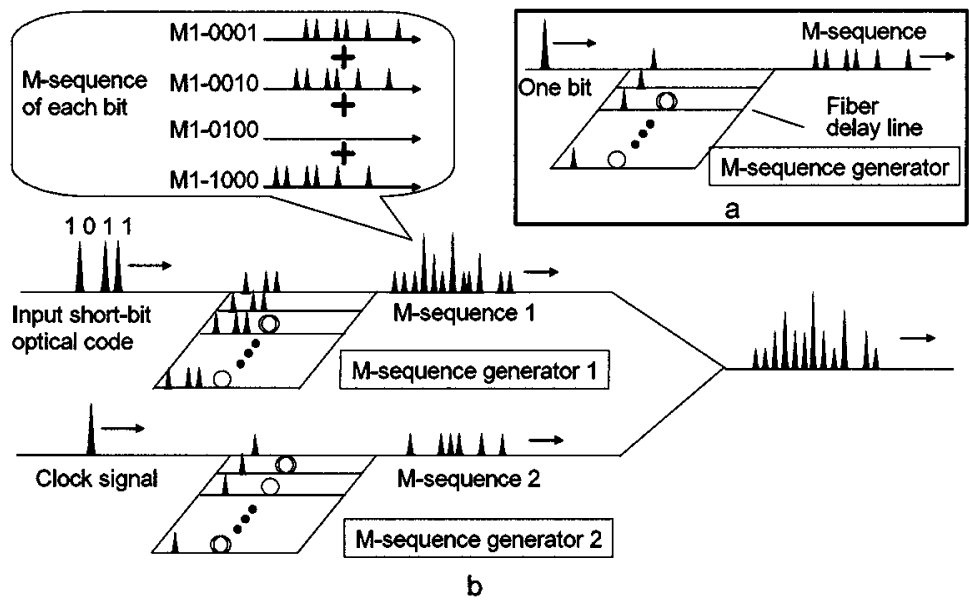

Figure 3. Front part of optical setup of spread coding system. (a) M-sequence generator.(b) Gold code generator.

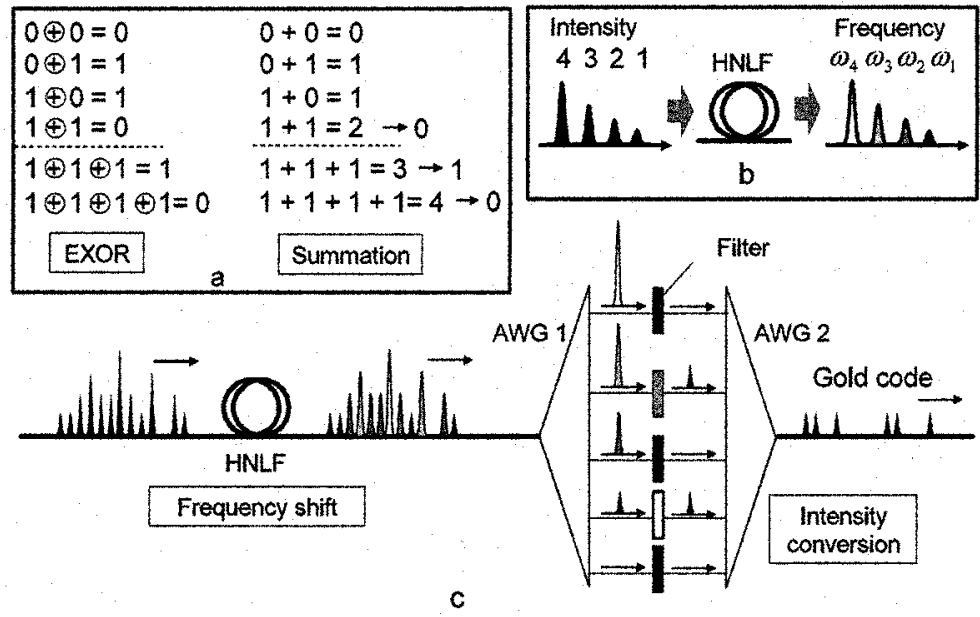

Figure 4. Rear part of optical setup of spread coding system. (a) EXOR operation and summation of light intensity. (b) Self-frequency shift in the HNLF. (c) EXOR operation using intensity conversion.

\section{SIMULATION OF LABEL RECOGNITION USING SPREAD CODING}

We calculate correlation signals of 16 different types of 127-bits BPSK Gold codes generated from 4-bits ASK optical codes. The Gold code generated from 
[1010] is set as the target Gold code. Figure 5 shows the maximum intensity of correlation signals of Gold codes with a matched filter. We can successfully obtain considerably high minimum SNR 15.2: 1 compared with SNR $8.0: 1$ in the previous work [4].

( G-0001: Gold code generated from [0001])

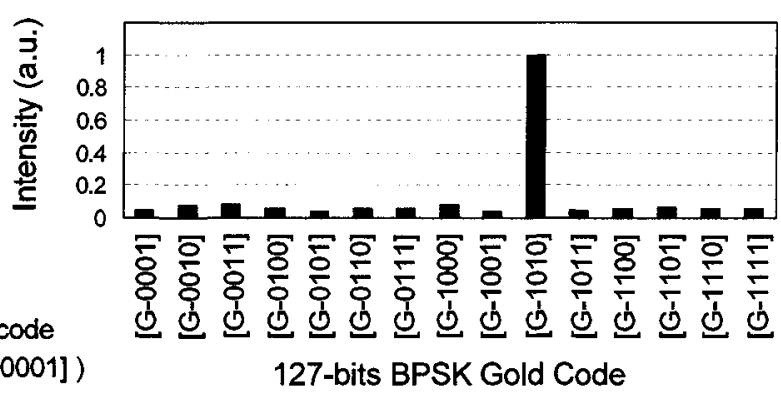

Figure 5. The maximum intensity of correlation signals of 127-bits Gold codes.

\section{CONCLUSION}

We proposed optical label recognition based on additional pre-spread coding, which can realize both high SNR and data transmission efficiency. From a calculation result, it is confirmed that high SNR can be realized using Gold codes generated by additional pre-spread coding.

\section{REFERENCES}

[1] Cardakli, M.C., Lee, S., Willner, A.E., Grubsky, V., Starodubov, D., Feinberg, J.: 'Reconfigurable optical packet header recognition and routing using time-to-wavelength mapping and tunable fiber Bragg gratings for correlation decoding', IEEE Photon. Tech. Lett., 2000, 12, (5), pp. 552-554.

[2] Kitayama, K., Wada, N., and Sotobayashi, H.: 'Architectural considerations for photonic IP router based upon optical code correlation', J.Lightwave Technol., 2000, 18, (12), pp. 1834-1844.

[3] Konishi, T., Kotanigawa, T., Tanimura, K., Furukawa, H., Oshita, Y., and Ichioka, Y.: 'Fundamental functions for ultrafast optical routing by temporal frequency-to-space conversion', Opt. Lett., 2001, 26, (18), pp. 1445-1447.

[4] Oshiba, S., Kutsuzawa, S., and Nishiki, A.: 'Optical label processing using a SSFBG encoder/decoder', IEICE Technol. Rep., 2003, PN 2003-15, (2003-09), pp. 29-34.

[5] Konishi, T., Tanimura, K., Asano, K., Oshita, Y., and Ichioka, Y.: 'All-optical analog-todigital converter by use of self-frequency shifting in fiber and a pulse-shaping technique', J. Opt. Soc. Am. B, 2002, 19, (11), pp. 2817-2823. 